\title{
Improved Ratio Type Estimators Using Auxiliary Attribute for Population Variance
}

\author{
Chandni Kumari ${ }^{1}$, Ratan Kumar Thakur ${ }^{2}$ \\ Department of Statistics, Babasaheb Bhimrao Ambedkar University, Lucknow-226025, India
}

\begin{abstract}
This paper proposes a family of estimators based on the auxiliary information on a attribute. The bias and mean squared error are obtained up to the first order of approximation. The theoretical comparison are also supported by numerical examples based on the two natural populations, showing the superiority of the suggested family of estimators, both theoretically as well as empirically over estimators available in literature.
\end{abstract}

Keywords: Ratio type estimator, bias, mean squared error, percent relative efficiency

\section{Introduction}

In sampling theory, the use of auxiliary information, is always beneficial in order to get more efficient estimates of the population parameters. Various authors have made the use of auxiliary attribute as a source of auxiliary information to increase the precision of the estimators, for the estimation of the population parameter under consideration. In recent years, many authors have also made use of various parameters associated with the auxiliary attribute $f$ for e.g., standard deviation $S_{f}$, coefficient of variation $C_{f}$, coefficient of kurtosis $b_{2}(f)$ and correlation coefficient $r$ of the population in estimation of the population variance. Bhushan (2013), Kalidar and Cingi (2003), Sisodia and Dwivedi (1981), Upadhyaya and Singh (1999), Singh et al. (2008), Pandey and Dubey (1988), etc are some of the authors in the list. In this paper, a family of estimators have been proposed by adapting the estimator of Koyuncu (2012) and a class of log type estimators (Kumari et al. (2019)) using the auxiliary information on a attribute. Consider a finite population $U=$ $\left(\mathrm{U}_{1}, \mathrm{U}_{2}, \ldots, \mathrm{U}_{\mathrm{N}}\right)$ of size $\mathrm{N}$ from which a sample of size $\mathrm{n}$ is drawn according to simple random sampling without replacement (SRSWOR). Let $y_{i}$ and $x_{i}$ denotes the values of the study variable and auxiliary attribute for the $\mathrm{i}^{\text {th }}$ unit $(\mathrm{i}=$ $1,2, \ldots, \mathrm{N})$, of the population. Further, let $\bar{y}$ and $\bar{f}$ be the sample means and $s_{y}^{2}=\frac{\sum_{i=1}^{n}(y i-\bar{y})^{2}}{(n-1)}$ and $s_{f}^{2}=\frac{\sum_{i=1}^{n}(f-\bar{f})^{2}}{(n-1)}$ be the sample variance of the study variable and auxiliary attribute respectively.

\section{Estimators Available in Literature}

\subsection{Conventional variance estimator}

$$
t_{0}=s_{y}^{2}
$$

The bias and variance of $t_{0}$ to the first order of approximation, are given as

$B\left(t_{0}\right)=0$

$V\left(t_{0}\right)=S_{y}^{4} I b_{2 y}^{*}$

\subsection{Isaki ratio estimator}

$$
t_{1}=s_{y}^{2}\left[\frac{s_{f}^{2}}{s_{f}^{2}}\right]
$$

The bias and MSE of $t_{1}$ to the first order of approximation, are given as

$$
\begin{aligned}
& B\left(t_{1}\right)=S_{y}^{2} \mathrm{I}\left[b_{2 f}^{*}-I_{22 y f}^{*}\right] \\
& V\left(t_{1}\right)=S_{y}^{4} \mathrm{I}\left[b_{2 y}^{*}+b_{2 f}^{*}-2 I_{22 y f}^{*}\right]
\end{aligned}
$$

\subsection{Conventional Product Estimator}

$$
t_{2}=s_{y}^{2}\left[\frac{s_{f}^{2}}{s_{f}^{2}}\right]
$$

The bias and MSE of $t_{2}$ to the first order of approximation, are given as

$$
\begin{aligned}
& B\left(t_{2}\right)=S_{y}^{2} \mathrm{I} b_{22}^{*} \\
& V\left(t_{2}\right)=S_{y}^{4} \mathrm{I}\left[b_{2 y}^{*}+b_{2 f}^{*}+2 I_{22 y f}^{*}\right]
\end{aligned}
$$

\subsection{Isaki regression estimator}

Isaki (1983) suggested the following regression estimator for population variance

$$
t_{3}=s_{y}^{2}+b\left(S_{f}^{2}-s_{f}^{2}\right)
$$

where $\mathrm{b}$ is a sample regression coefficient whose population regression coefficient is $\beta$.

The bias and MSE of $t_{3}$ to the first order of approximation, are given as

$$
\begin{aligned}
& B\left(t_{3}\right)=0 \\
& V\left(t_{3}\right)=S_{y}^{4} \mathrm{I}\left[b_{2 y}^{*}-\frac{I_{22 y f}^{* 2}}{b_{2 f}^{*}}\right]
\end{aligned}
$$

\subsection{Singh et al. estimator}

Singh et al. (1973) considered the following estimator,

$$
t_{4}=\alpha_{4} s_{y}^{2}
$$

Volume 9 Issue 4, April 2020

$$
\text { www.ijsr.net }
$$

Licensed Under Creative Commons Attribution CC BY 


\section{International Journal of Science and Research (IJSR) \\ ISSN: 2319-7064}

ResearchGate Impact Factor (2018): 0.28 | SJIF (2019): 7.583

where $\alpha_{4}$ is a Searl (1964) constant. The optimum value of Searls constant is $\alpha_{4}=\mathrm{n} /\left(\mathrm{n}+b_{2 y}^{*}\right)$ for which the mean squared error is minimum.

$\operatorname{MSE}\left(t_{4}\right)_{o p t}=S_{y}^{4} \mathrm{I}\left[\frac{n b_{2 y}^{*}}{\mathrm{n}+b_{2 y}^{*}}\right]$

\subsection{Das and Tripathi estimator}

$$
t_{5}=s_{y}^{2}\left[\frac{S_{f}^{2}}{S_{f}^{2}+\alpha_{5}\left(s_{f}^{2}-S_{f}^{2}\right)}\right]
$$

where $\alpha_{5}$ is a constant. The bias and MSE of $t_{5}$ to the first degree of approximation is given as

$$
\begin{gathered}
B\left(t_{5}\right)=S_{y}^{2} \mathrm{I}\left[\alpha_{5} b_{2 y}^{*}-\alpha_{5} I_{22 y f}^{*}\right] \\
V\left(t_{5}\right)=S_{y}^{4} \mathrm{I}\left[b_{2 y}^{*}+\alpha_{5} b_{2 f}^{*}-2 \alpha_{5} I_{22 y f}^{*}\right]
\end{gathered}
$$

The MSE of $t_{5}$ is optimum for $\alpha_{5}=\frac{I_{22 y f}^{*}}{b_{2 f}^{*}}$ and is given by

$$
\operatorname{MSE}\left(t_{5}\right)_{o p t}=S_{y}^{4} I\left[b_{2 y}^{*}-I_{22 y f}^{* 2} / b_{2 f}^{*}\right]
$$

\subsection{Prasad and Singh estimator}

Prasad and Singh (1992) introduced the following estimator

$t_{6}=\alpha_{6} s_{y}^{2}\left[\frac{S_{f}^{2}}{S_{f}^{2}}\right]$

where $\alpha_{6}$ is a constant. The bias and MSE of $t_{6}$ to the first degree of approximation is given as

$$
\begin{aligned}
& B\left(t_{6}\right)=S_{y}^{2} \mathrm{I}\left[\alpha_{6}\left(n+b_{2 f}^{*}-I_{22 y f}^{*}\right)-n\right] \\
& V\left(t_{6}\right)=S_{y}^{4} \mathrm{I}\left[\alpha_{6}^{2}\left(n+b_{2 y}^{*}+3 b_{2 f}^{*}-4 I_{22 y f}^{*}\right)-\right. \\
& 2 \alpha 6 n+b 2 f *-I 22 y f *-n
\end{aligned}
$$

The MSE of $t_{6}$ is optimum for

$\alpha_{6}=\left(n+b_{2 f}^{*}-I_{22 y f}^{*}\right) /\left(n+b_{2 y}^{*}+3 b_{2 f}^{*}-4 I_{22 y f}^{*}\right)$

and is given by

$\operatorname{MSE}\left(t_{6}\right)_{o p t}=S_{y}^{4} I\left[n-\frac{\left(n+b_{2 f}^{*}-I_{22 y f}^{*}\right)^{2}}{\left(n+b_{2 y}^{*}+3 b_{2 f}^{*}-4 I_{22 y f}^{*}\right)}\right]$

\subsection{Garcia and Cebrian estimator}

Garcia and Cebrian (1996) introduced the following estimator

$$
t_{7}=s_{y}^{2}\left[\frac{S_{f}^{2}}{S_{f}^{2}}\right]^{\alpha_{7}}
$$

where $\alpha_{7}$ is a constant. The bias and MSE of $t_{6}$ to the first degree of approximation is given as

$$
\begin{aligned}
& B\left(t_{7}\right)=S_{y}^{2} \mathrm{I}\left[\alpha_{7} \frac{\left(\alpha_{7}+1\right)}{2} b_{2 f}^{*}-\alpha_{7} I_{22 y f}^{*}\right] \\
& V\left(t_{7}\right)=S_{y}^{4} \mathrm{I}\left[b_{2 y}^{*}+\alpha_{7}^{2} b_{2 f}^{*}-2 \alpha_{7} I_{22 y f}^{*}\right]
\end{aligned}
$$

The MSE of $t_{7}$ is optimum for $\alpha_{7}=\frac{I_{22}^{*}}{b_{2 f}^{*}}$ and is given by

$$
\operatorname{MSE}\left(t_{7}\right)_{o p t}=S_{y}^{4} I\left[b_{2 y}^{*}-I_{22 y f}^{* 2} / b_{2 f}^{*}\right]
$$

\subsection{Upadhaya and Singh estimator}

Upadhaya and Singh (2001) suggested following estimator

$t_{8}=s_{y}^{2}+\alpha_{8}\left(s_{f}^{2}-s_{f}^{2}\right)$

where $\alpha_{8}$ is a constant. The MSE of $t_{8}$ is optimum for $\alpha_{8}=\frac{S_{y}^{2} I_{22 y f}^{*}}{S_{f}^{2} b_{2 f}^{*}}$ and is given by

$$
\operatorname{MSE}\left(t_{8}\right)_{o p t}=S_{y}^{4} I\left[b_{2 y}^{*}-I_{22 y f}^{* 2} / b_{2 f}^{*}\right]
$$

\subsection{Shabbir and Gupta (2006) estimator}

Sabbir and Gupta (2006) proposed the following estimator $t_{9}=\lambda t_{m}$

where $\lambda$ is a Searls (1964) contant whose value is to be determined later. Here $t_{m}$ is a combination of Singh et al. (1973), Prasad and Singh (1992) and is defined as

$t_{m}=K_{1} s_{y}^{2}+K_{2} s_{y}^{2}\left(\frac{S_{f}^{2}}{s_{f}^{2}}\right)$

where $K_{1}$ and $K_{2}$ are the weights such that $K_{1}+K_{2}=1$

The optimum MSE of $t_{9}$ is given by

$$
\operatorname{MSE}\left(t_{9}\right)_{o p t}=S_{y}^{4} I\left[n-\frac{\left(n+I_{22 y f}^{*}-\frac{I_{22 y f}^{* 2}}{b_{2 f}^{*}}\right)^{2}}{\left(n+b_{2 y}^{*}+2 I_{22 y f}^{*}-3 \frac{I_{22 y f}^{* 2}}{b_{2 f}^{*}}\right)}\right]
$$

\subsection{Shabbir and Gupta (2007) estimator}

$t_{10}=K_{1} s_{y}^{2}+K_{2}\left(S_{f}^{2}-s_{f}^{2}\right) \exp \left(\frac{S_{f}^{2}-s_{f}^{2}}{S_{f}^{2}+s_{f}^{2}}\right)$

where $K_{1}$ and $K_{2}$ are suitably chosen coantants.

Situation 1. $\mathrm{k} 1+K_{2}=1$ The bias and MSE of $t_{10}$ the first degree of approximation are given as

Volume 9 Issue 4, April 2020 


\section{International Journal of Science and Research (IJSR) \\ ISSN: 2319-7064}

ResearchGate Impact Factor (2018): 0.28 | SJIF (2019): 7.583

The optimum MSE of $t_{10}$ is given by

$$
\operatorname{MSE}\left(t_{10}\right)_{o p t}=S_{y}^{4} I\left[A_{1}-\frac{\left(A_{1}+A_{3}\right)^{2}}{\left(A_{1}+A_{3}+2 A_{3}\right)}\right]
$$

Situation 2. Unconstrained choice of $K_{1}$ and $K_{2}$ The bias and MSE of $t_{10}$ the first degree of approximation are given as

$$
\operatorname{MSE}\left(t_{10}^{*}\right)_{o p t}=S_{y}^{4} I\left[\frac{\operatorname{Var}\left(\hat{S}_{r e g}\right)}{1+\frac{\operatorname{Var}\left(\hat{S}_{r e g}\right)}{S_{y}^{4}}}\right]
$$

\subsection{Kadilar and Cingi estimator}

Kadilar and Cingi (2006) suggested the following ratio type estimator

$t_{11}=w_{1} s_{y}^{2}+K_{2}\left(s_{y}^{2} \frac{s_{f}^{2}}{s_{f}^{2}}\right) v$

where $w_{1}$ and $w_{2}$ are the weights such that $w_{1}+w_{2}=1$

The optimum MSE of $t_{11}$ is given by

$\operatorname{MSE}\left(t_{11}\right)_{o p t}=S_{y}^{4} I\left[n-\frac{\left(n+I_{22 y f}^{*}-\frac{I_{22 y f}^{* 2}}{b_{2 f}^{*}}\right)^{2}}{\left(n+b_{2 y}^{*}+2 I_{22 y f}^{*}-3 \frac{I_{22 y f}^{* 2}}{b_{2 f}^{*}}\right)}\right]$

\subsection{Yadav and Kalidar (2013) estimator}

Yadav and Kalidar (2013) introduced the following estimator

$t_{12}=s_{y}^{2}+\exp \left(1-\frac{\alpha_{12} s_{f}^{2}}{S_{f}^{2}+\left(\alpha_{12}-1\right) s_{f}^{2}}\right)$

Where $\alpha_{12}$ is a constant. The optimum MSE of $t_{12}$ is given by

\section{Properties of the Suggested Classes of Log-Type Estimators}

In order to obtain the bias and mean square error (MSE), let us consider

$E\left(\epsilon_{0}\right)=E\left(\epsilon_{1}\right)=0, E\left(\epsilon_{0}\right)^{2}=I b_{2 y}^{*}, E\left(\epsilon_{1}\right)^{2}=I b_{2 f}^{*}, E\left(\epsilon_{0} \epsilon_{1}\right)=I I_{22 y f}^{*}$,

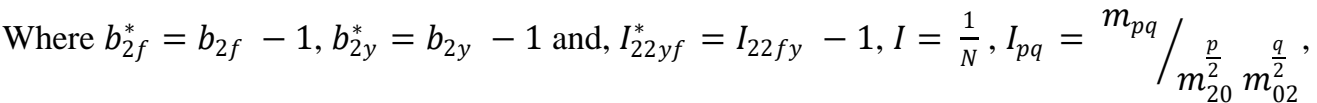

$m_{p q}=\sum_{i=1}^{N}\left(Y_{i}-\bar{Y}\right)^{P}\left(f_{i}-\bar{f}\right)^{q} /{ }_{N}, b_{2 y}=m_{40} /_{m_{20}^{2}}, b_{2 f}=m_{04} / m_{02}^{2}$ are the coefficient of kurtosis of $y$ and $f$ respectively. 


\section{International Journal of Science and Research (IJSR)}

ISSN: 2319-7064

ResearchGate Impact Factor (2018): 0.28 | SJIF (2019): 7.583

Theorem 1. The bias and mean squared error of the proposed estimators are given by

$\operatorname{Bias}(T)=S_{y}^{2}\left[w_{1}\left(1+I a \eta r_{y f} \sqrt{b_{2 y}^{*} b_{2 f}^{*}}-\frac{a \eta^{2}}{2} I b_{2 f}^{*}\right)-1\right]+w_{2}\left(1+I a \eta b_{2 f}^{*}-\frac{a \eta^{2}}{2} I b_{2 f}^{*}\right)$

$\operatorname{MSE}(T)=S_{y}^{4} w_{1}^{2} A+w_{2}^{2} B+S_{y}^{4} w_{1} D+S_{y}^{2} w_{2} G+S_{y}^{2} w_{1} w_{2} F+S_{y}^{4} \quad$ where

$A=\left[1+I\left(b_{2 y}^{*}+a^{2} \eta^{2} b_{2 f}^{*}+4 a \eta r_{y f} \sqrt{b_{2 y}^{*} b_{2 f}^{*}}-a \eta^{2} b_{2 f}^{*}\right)\right]$

$B=\left[1+I\left(b_{2 f}^{*}+a^{2} \eta^{2} b_{2 f}^{*}+4 a \eta r_{y f} b_{2 f}^{*}-a \eta^{2} b_{2 f}^{*}\right)\right]$

$D=I\left(a \eta^{2} b_{2 f}^{*}-2 a \eta r_{y f} \sqrt{b_{2 y}^{*} b_{2 f}^{*}}\right)-2$

$G=I\left(a \eta^{2} b_{2 f}^{*}-2 a \eta b_{2 f}^{*}\right)-2$

$F=2+2 I\left(2 a \eta b_{2 f}^{*}+2 a \eta r_{y f} \sqrt{b_{2 y}^{*} b_{2 f}^{*}}+r_{y f} \sqrt{b_{2 y}^{*} b_{2 f}^{*}}-a \eta^{2} b_{2 f}^{*}+a^{2} \eta^{2} b_{2 f}^{*}\right)$

$\eta=\frac{a S_{f}^{2}}{a S_{f}^{2}+b}, r_{y f}=\frac{I_{22 y f}^{*}}{\sqrt{b_{2 y}^{*} b_{2 f}^{*}}}$

respectively,

Corollary 1- The mean square error of the proposed class of estimator $T_{c}$ will be minimum for the optimum value of the characterizing parameters, given

$w_{1 o p t}=\frac{G F-2 B D}{4 A B-F^{2}}$
$w_{2 o p t}=S_{y}^{2}\left[\frac{D F-2 G A}{4 A B-F^{2}}\right]$

and the minimum value of the mean square error within the proposed class of estimator is

$\operatorname{MSE}\left(T_{c}\right)_{o p t}=S_{y}^{4}\left[1-\frac{B D^{2}-D F G+G^{2} A}{4 A B-F^{2}}\right]$

\section{Multivariate extension of the suggested classes of estimators using multiple auxiliary information}

Let there are k auxiliary attributes then we can use the attributes by taking a linear combination of these k estimators of the form given in section 2, calculated for every auxiliary attribute separately, for estimating the population variance. Then the estimators for population variance will be defined as

$$
T_{c}=\left[w_{1} s_{y}^{2}+w_{2}\left(\frac{S_{f}^{2}}{s_{f}^{2}}\right)\right] \prod_{i=1}^{n}\left[1+a_{i} \log \left(\frac{S_{f_{i}}^{* 2}}{s_{f_{i}}^{* 2}}\right)\right]
$$

where $a_{i}{ }^{\prime} s$ are the optimizing scalar, $\mathrm{i}=1,2, \ldots \mathrm{n}$.

\section{Properties of the suggested classes of estimators using multiple auxiliary information}

Theorem 3. The bias of the proposed estimators are given as

$$
\begin{aligned}
& \operatorname{Bias}(T)=S_{y}^{2}\left[w_{1}\left(1+I \sum_{i=1}^{n} a_{i} \eta^{2} b_{2 f_{i}}^{*}-I \sum_{i=1}^{n} a_{i} \eta r_{y f i} \sqrt{b_{2 y}^{*} b_{2 f_{i}}^{*}}-I \sum_{i=1}^{n} \frac{a_{i}{ }^{2} \eta^{2}}{2} b_{2 f_{i}}^{*}\right)-1\right] \\
& +w_{2}\left(1+I b_{2 f_{i}}^{*}+I \sum_{i=1}^{n} a_{i} \eta b_{2 f_{i}}^{*}+I \sum_{i=1}^{n} \frac{a_{i}{ }^{2} \eta^{2}}{2} b_{2 f_{i}}^{*}\right) \\
& \operatorname{MSE}(T)=S_{y}^{4} w_{1}^{2} A+w_{2}^{2} B+S_{y}^{4} w_{1} D+S_{y}^{2} w_{2} G+S_{y}^{2} w_{1} w_{2} F+S_{y}^{4}
\end{aligned}
$$

where

$$
A=\left[1+I\left(b_{2 y}^{*}+\sum_{i=1}^{n} a_{i}^{2} \eta^{2} b_{2 f_{i}}^{*}-4 \sum_{i=1}^{n} a_{i} \eta r_{y f_{i}} \sqrt{b_{2 y}^{*} b_{2 f_{i}}^{*}}-\sum_{i=1}^{n} a_{i} \eta^{2} b_{2 f_{i}}^{*}\right)\right]
$$




\section{International Journal of Science and Research (IJSR)}

ISSN: 2319-7064

ResearchGate Impact Factor (2018): 0.28 | SJIF (2019): 7.583

$$
\begin{aligned}
& B=\left[1+I\left(b_{2 f_{i}}^{*}+\sum_{i=1}^{n} a_{i}^{2} \eta^{2} b_{2 f_{i}}^{*}+4 \sum_{i=1}^{n} a_{i} \eta r_{y f_{i}} b_{2 f_{i}}^{*}+\sum_{i=1}^{n} a_{i} \eta^{2} b_{2 f_{i}}^{*}\right)\right] \\
& D=I\left(2 \sum_{i=1}^{n} a_{i} \eta r_{y f_{i}} \sqrt{b_{2 y}^{*} b_{2 f_{i}}^{*}}-\sum_{i=1}^{n} a_{i} \eta^{2} b_{2 f_{i}}^{*}\right)-2 \\
& G=I\left(-\sum_{i=1}^{n} a_{i} \eta^{2} b_{2 f_{i}}^{*}-2 \sum_{i=1}^{n} a_{i} \eta b_{2 f_{i}}^{*}\right)-2 \\
& F=2+2 I\left(2 \sum_{i=1}^{n} a_{i} \eta b_{2 f_{i}}^{*}-2 \sum_{i=1}^{n} a_{i} \eta r_{y f_{i}} \sqrt{b_{2 y}^{*} b_{2 f_{i}}^{*}}-r_{y f_{i}} \sqrt{b_{2 y}^{*} b_{2 f_{i}}^{*}}+\sum_{i=1}^{n} a_{i} \eta^{2} b_{2 f_{i}}^{*}+\sum_{i=1}^{n} a_{i} \eta^{2} b_{2 f_{i}}^{*}\right) \\
& \eta=\frac{a S_{f_{i}}^{2}}{a S_{f_{i}}^{2}+b}, r=\frac{I_{22 y f_{i}}^{*}}{\sqrt{b_{2 y}^{*} b_{2 f_{i}}^{*}}} \text { respectively. }
\end{aligned}
$$

\section{Some members of the class of estimators $\mathbf{T}_{c}$}

It can be easily seen that the proposed class $T_{c}$ is a generalized form of class of estimators for the $a_{1}(\neq 0), b 1$ and $a_{2}(\neq 0), \quad b_{2}$ are either real numbers or functions of the known parameters of the auxiliary attribute $f$ such as the standard deviations $\mathrm{S}_{\mathrm{f}}$, coefficient of variation $\mathrm{C}_{\mathrm{f}}$, coefficient of kurtosis $b_{2}(f)$ coefficient of skewness $b_{1}(f)$ and correlation coefficient $r$ of the population. Therefore, a wide variety of estimators can be designed using the above known population parameters. Some of them are listed below.

Table 1: Some generalized members of the proposed class of estimators $T_{c}$

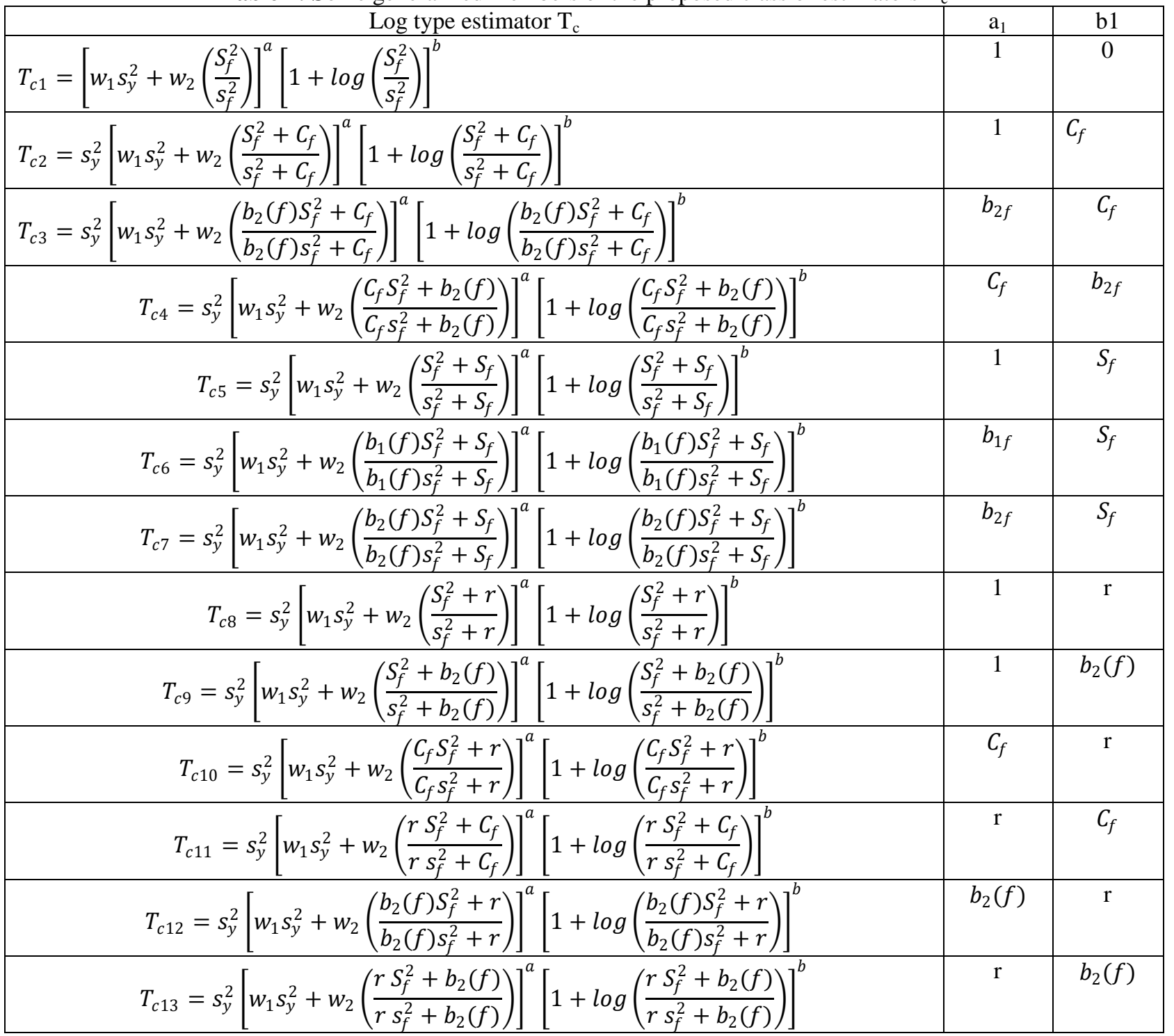

\section{Volume 9 Issue 4, April 2020} www.ijsr.net 


\section{International Journal of Science and Research (IJSR) \\ ISSN: 2319-7064}

ResearchGate Impact Factor (2018): 0.28 | SJIF (2019): 7.583

\section{Comparison of estimators}

In this section, we compare the proposed classes of estimators with some important estimators. The comparison will be in terms of their MSEs up to the order of $n^{-1}$. Let us define

$C_{1}=b_{2 y}^{*}+b_{2 f}^{*}-2 I_{22}^{*}, C_{2}=b_{2 y}^{*}+b_{2 f}^{*}-2 I_{22}^{*}$,

$\mathrm{D}=b_{2 y}^{*} b_{2 f}^{*}-I_{22}^{*}, \quad \mathrm{E}=\frac{n b_{2 y}^{*}}{n+b_{2 y}^{*}}$

$\mathrm{F}=\left[n-\frac{\left(n+b_{2 f}^{*}-I_{22}^{*}\right)^{2}}{n+b_{2 y}^{*}+3 b_{2 f}^{*}-4 I_{22}^{*}}\right], \mathrm{G}=\left[n-\frac{\left(n+I_{22}^{*}-\frac{I_{22}^{*}}{b_{2 f}^{*}}\right)^{2}}{n+b_{2 y}^{*}+2 I_{22}^{*}-3 \frac{I_{22}^{*}}{b_{2 f}^{*}}}\right]$,

$\mathrm{H}=\left[A_{1}-\frac{\left(A_{1}+A_{3}\right)^{2}}{A_{1}+A_{2}+2 A_{3}}\right]$

$\operatorname{MSE}\left(t_{0}\right)>\operatorname{MSE}\left(T_{1}^{*}\right)_{\text {opt }} \quad$ if $b_{2 y}^{*}+\frac{B^{2}}{A}-\mathrm{n}>0$

$\operatorname{MSE}\left(t_{1}\right)>\operatorname{MSE}\left(T_{1}^{*}\right)_{o p t} \quad$ if $C_{1}+\frac{B^{2}}{A}-\mathrm{n}>0$

$\operatorname{MSE}\left(t_{2}\right)>\operatorname{MSE}\left(T_{1}^{*}\right)_{\text {opt }} \quad$ if $\quad C_{2}+\frac{B^{2}}{A}-\mathrm{n}>0$

$\operatorname{MSE}\left(t_{3}\right)>\operatorname{MSE}\left(T_{1}^{*}\right)_{o p t} \quad$ if $\quad D-\left(n-\frac{B^{2}}{A}\right) b_{2 f}^{*}>0$

$\operatorname{MSE}\left(t_{4}\right)>\operatorname{MSE}\left(T_{1}^{*}\right)_{\text {opt }}$ if $E-\frac{B^{2}}{A}-\mathrm{n}>0$

$\operatorname{MSE}\left(t_{5}\right)>\operatorname{MSE}\left(T_{1}^{*}\right)_{\text {opt }} \quad$ if $\quad D-\left(n-\frac{B^{2}}{A}\right) b_{2 f}^{*}>0$

$\operatorname{MSE}\left(t_{6}\right)>\operatorname{MSE}\left(T_{1}^{*}\right)_{o p t} \quad$ if $\quad F+\frac{B^{2}}{A}-\mathrm{n}>0$

$\operatorname{MSE}\left(t_{7}\right)>\operatorname{MSE}\left(T_{1}^{*}\right)_{\text {opt }} \quad$ if $\quad D-\left(n-\frac{B^{2}}{A}\right) b_{2 f}^{*}>0$

$\operatorname{MSE}\left(t_{8}\right)>\operatorname{MSE}\left(T_{1}^{*}\right)_{\text {opt }} \quad$ if $\quad D-\left(n-\frac{B^{2}}{A}\right) b_{2 f}^{*}>0$

$\operatorname{MSE}\left(t_{9}\right)>\operatorname{MSE}\left(T_{1}^{*}\right)_{\text {opt }}$ if $\quad G+\frac{B^{2}}{A}-\mathrm{n}>0$

$\operatorname{MSE}\left(t_{10}\right)>\operatorname{MSE}\left(T_{1}^{*}\right)_{o p t}$ if $H+\frac{B^{2}}{A}-\mathrm{n}>0$

$\operatorname{MSE}\left(t_{11}\right)>\operatorname{MSE}\left(T_{1}^{*}\right)_{o p t} \quad$ if $\quad G+\frac{B^{2}}{A}-\mathrm{n}>0$

$\operatorname{MSE}\left(t_{12}\right)>\operatorname{MSE}\left(T_{1}^{*}\right)_{\text {opt }} \quad$ if $D-\left(n-\frac{B^{2}}{A}\right) b_{2 f}^{*}>0$

\section{Empirical Study}

To compare the efficiency of the suggested class of estimator numerically, we considered nine natural data sets. The description of the population is given below.

Population 1. (Cochran (1977), Pg. no. 107)

$\mathrm{y}$ : number of persons per block

$\mathrm{f}$ : number of rooms per block

$S_{y}^{2}=214.69, S_{f}^{2}=56.76, b_{2 y}^{*}=1.2387, b_{2 f}^{*}=1.3523, I_{22}^{*}=$ $0.5432, \mathrm{C}_{\mathrm{f}}=0.1450, \bar{f}=58.8$,

$\rho=0.6515, \mathrm{n}=10$.
Population 2. (Cochran (1977), Pg. no. 203)

$\mathrm{y}:$ actual weight of peaches on each tree

$\mathrm{f}$ : eye estimate of weight of peaches on each tree.

$S_{y}^{2}=99.81, S_{f}^{2}=85.09, b_{2 y}^{*}=0.9249, b_{2 f}^{*}=1.2932, I_{22}^{*}=$ $1.1149, \mathrm{C}_{\mathrm{f}}=0.1621, \bar{f}=56.9$,

$\rho=0.9937, \mathrm{n}=10$.

Population 3. (Sukhatme P. V. (1970), Pg. no. 185)

$\mathrm{y}$ : wheat acreage in 1937

$\mathrm{f}:$ wheat acreage in 1936

$S_{y}^{2}=26456.99, S_{f}^{2}=22355.76, b_{2 y}^{*}=2.1842, b_{2 f}^{*}=1.2030$,

$I_{22}^{*}=1.5597, \mathrm{C}_{\mathrm{f}}=0.5625, \bar{f}=265.8, \rho=0.977, \mathrm{n}=10$.

Population 4. (Singh D and Chaudhary F. S., Pg. no. 107). $\mathrm{y}:$ number of boats landing at a particular centre

$\mathrm{f}$ : catch of fish in quintals.

$S_{y}^{2}=201324.4, S_{f}^{2}=396.8889, b_{2 y}^{*}=0.9462, b_{2 f}^{*}=0.6078$,

$I_{22}^{*}=0.6333, \mathrm{C}_{\mathrm{f}}=0.7288$,

$\bar{f}=27.3333, \rho=0.9308, \mathrm{n}=4$.

Population 5. (Singh D and Chaudhary F. S., Pg. no. 141). $\mathrm{y}$ :number of bearing lime trees

$\mathrm{f}$ : area under lime (in acres)

$S_{y}^{2}=6564586.45, S_{f}^{2}=1092.1024, b_{2 y}^{*}=12.2574, b_{2 f}^{*}=4.5788$,

$I_{22}^{*}=6.7126, \mathrm{C}_{\mathrm{f}}=1.4273$,

$\bar{f}=22.6209, \rho=0.9021, \mathrm{n}=9$.

Population 6. (Choudhary F. S. and Singh D., Pg. no. 176). $\mathrm{y}:$ number of cows in milk enumerated

$\mathrm{f}$ : number of cows in milk in the previous year.

$S_{y}^{2}=332721.2079, \quad S_{f}^{2}=281472.7868, \quad b_{2 y}^{*}=6.2079$,

$b_{2 f}^{*}=5.0043, I_{22}^{*}=4.9528, \mathrm{C}_{\mathrm{f}}=0.8276$,

$\bar{f}=641.05, \rho=0.8933, \mathrm{n}=8$.

Population 7. (Singh S., Pg. no. 324-325).

$\mathrm{y}:$ approximate duration of sleep (in minutes)

$\mathrm{f}:$ age in years of the persons.

$S_{y}^{2}=3582.579, S_{f}^{2}=85.2367, b_{2 y}^{*}=1.6678, b_{2 f}^{*}=1.2389$,

$I_{22}^{*}=0.9961, \mathrm{C}_{\mathrm{f}}=0.1349, \bar{f}=67.2667$,

$\rho=-0.8552, \mathrm{n}=9$.

Population 8. (Singh S., Pg. no. 1114).

$\mathrm{y}:$ appropriate duration of sleep (in minutes)

$\mathrm{f}:$ age in years of the persons.

$S_{y}^{2}=0.0073, S_{f}^{2}=0.0063, b_{2 y}^{*}=2.6323, b_{2 f}^{*}=2.4016$,

$I_{22}^{*}=1.8351, \mathrm{C}_{\mathrm{f}}=1.2352, \bar{f}=0.1831, \rho=0.7789, \mathrm{n}=11$.

By using the above data set, the percent relative efficiency of the different estimator are given in Table 2. 
International Journal of Science and Research (IJSR)

ISSN: 2319-7064

ResearchGate Impact Factor (2018): 0.28 | SJIF (2019): 7.583

\begin{tabular}{|c|c|c|c|c|c|c|c|c|}
\hline Est. & Pop 1 & Pop 2 & Pop 3 & Pop 4 & Pop 5 & Pop 6 & Pop 7 & Pop 8 \\
\hline$t_{0}$ & 100 & 100 & 100 & 100 & 100 & 100 & 100 & 100 \\
\hline$t_{1}$ & 121.38 & 320.81 & 815.60 & 329.16 & 359.35 & 475.29 & 182.39 & 193.03 \\
\hline$t_{2}$ & 33.68 & 19.48 & 33.56 & 33.54 & 40.50 & 29.39 & 34.04 & 30.24 \\
\hline$t_{3}$ & 121.38 & 639.14 & 1347.98 & 330.39 & 507.23 & 475.29 & 192.40 & 214.00 \\
\hline$t_{4}$ & 112.38 & 639.14 & 121.42 & 30.91 & 236.19 & 177.59 & 118.53 & 123.93 \\
\hline$t_{5}$ & 121.38 & 109.23 & 1347.98 & 330.96 & 507.23 & 475.29 & 192.40 & 214.00 \\
\hline$t_{6}$ & 112.95 & 639.14 & 818.13 & 348.83 & 381.77 & 558.96 & 212.28 & 242.02 \\
\hline$t_{7}$ & 121.38 & 639.14 & 1347.98 & 330.39 & 507.23 & 475.29 & 192.40 & 214.00 \\
\hline$t_{8}$ & 121.38 & 639.14 & 1347.98 & 330.39 & 507.23 & 475.29 & 192.40 & 214.03 \\
\hline$t_{9}$ & 143.14 & 749.18 & 1434.48 & 349.88 & 528.70 & 559.08 & 220.35 & 258.35 \\
\hline$t_{10}$ & 121.38 & 489.41 & 306.84 & 227.25 & 319.46 & 457.77 & 188.82 & 228.88 \\
\hline$t_{10}^{*}$ & 82.34 & 648.39 & 1369.81 & 354.05 & 643.42 & 552.89 & 210.93 & 237.93 \\
\hline$t_{11}$ & 82.53 & 322.84 & 815.55 & 329.05 & 358.77 & 269.13 & 182.54 & 102.02 \\
\hline$t_{12}$ & 121.38 & 639.14 & 1347.98 & 330.39 & 507.23 & 475.29 & 192.40 & 214.00 \\
\hline$t_{\alpha, \beta_{12}}$ & 100 & 100 & 100 & 100 & 100 & 100 & 100 & 100 \\
\hline$T_{c}$ & 147.02 & 1163.41 & 1863.95 & 439.47 & 991.93 & 2120.43 & 247.66 & 311.67 \\
\hline
\end{tabular}

In the above table, the relative efficiency of the proposed estimator is much better as compared to other estimators for all the data sets given here.

\section{Conclusion}

The present study extends the idea of Kumari et al. (2019) regarding the effective use of auxiliary information if the relationship between the study variable and the auxiliary attribute is of logarithmic type. Further, the efficiency of the proposed estimators are compared with some conventional estimators and some recent estimators of Singh et al. (1973), Das and Tripathi (1978), Sisodia and Dwivedi (1981), Isaki (1983), Bahl and Tuteja (1991), Prasad and Singh (1992), Swain (1994), Garcia and Cebrian (1996), Upadhaya and Singh (2001), Kalidar and Cingi (2006a, 2006b); Gupta and Shabbir (2006, 2007), Yadav and Kadilar (2013, 2014). The proposed estimator is most efficient than all the estimators. This study is also supported through an empirical sudy and the result of this study is quite encouraging.

\section{References}

[1] Kumari, C., Bhushan, S. And Thakur, R. K. (2019). Optimal Two Parameter Logarithmic Estimators for Estimating the Population Variance, Global Journal of Pure and Applied Mathematics, Volume 15, Issue 5, pg 527-237.

[2] Bhushan, S. and Kumari, C (2019). Double Sampling Log-type Estimator Using Auxiliary Attribute for Population Variance, Journal of Statistics Applications \& Probability, 6, No. 3, pg 1-6.

[3] Bhushan, S. and Kumari, C. (2018). A new log type estimators for estimating the population variance, International Journal of Computational and Applied Mathematics (ISSN 1819-4966), 13 (1), 43-54.

[4] Bhushan, S. and Kumari, C. (2018). A Class of Double Sampling Log-Type Estimators for Population Variance Using Two Auxiliary Variable, International Journal of Applied Engineering Research, Volume 13, Number (13) 2018, pg 11151-11155.

[5] Bhushan, S. and Kumari, C. (2018). Some Classes of Log Type Estimators Using Auxiliary Attribute for Population Variance, International Journal of Scientific and Engineering Research, Volume 9, Issue 6, pg 1823-1832.

[6] Bhushan, S. and Kumari, C. (2018). Estimation of Variance of Finite Population Using Double Sampling Scheme, International Journal of Scientific and Engineering Research, Volume 9, Issue 8, pg 18931901.

[7] Kumari, C., Bhushan, S. and Thakur, R. K. (2018) Modied Ratio Estimators Using Two Auxiliary Information for Estimating Population Variance in Two-Phase Sampling, International Journal of Scientific and Engineering Research, Volume 9, Issue 8,pg 1884- 1892 .

[8] Bahl S. and Tuteja R. K. (1991). Ratio and Product type exponential estimator, Info. Optim. Sci., Vol. XII(I), 159-163.

[9] Bhushan S., Gupta R. and Pandey S. K. (2015). Some log-type classes of estimators using auxiliary information.

[10] Hidiroglou M. A. and Sarndal C. E. (1998). Use of auxiliary information for two-phase sampling, Survey Methodology, 24(1), 11-20.

[11] Neyman J. (1938). Contribution to the theory of sampling human populations, J. Amer. Stat. Asso., 33, 101-116.

[12] Cochran W. G. (1963). Sampling Techniques, Wiley Eastern Private Limited, New Delhi, 307-310.

[13] Chaudhury A. (1978). On estimating the variance of a finite population. Metrika, 25, 66-67.

[14] Das A. K. and Tripathi T. P. (1978). Use of auxiliary information in estimating the nite population variance. Sankhya, C (4), 139-148.

[15] Gupta S. and Shabbir J. (2008). Variance estimation in simple random sampling using auxiliary information, Hacettepe Journal of Mathematics and Statistics, 37, 57-67.

[16] Isaki C. T. (1983). Variance estimation using Auxiliary Information, Jour. Amer. Statist. Asssoct, 78, 117-123.

[17] Kadilar C. and Cingi H. (2006)a. Improvement in variance estimation using auxiliary information, Hacettepe Journal of Mathematics and Statistics, 1(35), 111-115.

[18] Kadilar C. and Cingi H. (2006)b. Ratio estimators for population variance in simple and stratied sampling, Applied Mathematics and Computation, 1(73), 10471058.

\section{Volume 9 Issue 4, April 2020}




\section{International Journal of Science and Research (IJSR) \\ ISSN: 2319-7064}

ResearchGate Impact Factor (2018): 0.28 | SJIF (2019): 7.583

[19] Sukhatme P. V., Sukhatme B. V., Sukhatme S. and Ashok C. (1984). Sampling Theory of Surveys with Applications, Iowa State University Press, Ams.

[20] Singh, R., Chauhan, P., Sawan, N. \& Smarandache, F. (2011), Improved exponential estimator for population variance using two auxiliary variables, Ital. J. Pure Appl. Math.s 28, 101108.

[21] Swain A. K. P. C. and Mishra G. (1994). Estimation of population variance under unequal probability sampling, Sankhya, B (56), 374-384.

[22] Bhushan S., Misra P. K. and Yadav S. K. (2017b). A Generalized Class of Unbiased Estimators for Population Mean Using Auxiliary Information on an Attribute and an Auxiliary Variable, Int. J. Comp. App. Math., 12(1), 11-28.

Volume 9 Issue 4, April 2020 www.ijsr.net 